\title{
Editorial
}

\section{Advances in Massive MIMO Antenna Design, Channel Modeling, and System Technologies}

\author{
Kan Zheng, ${ }^{1}$ Xuefeng Yin, ${ }^{2}$ Periklis Chatzimisios, ${ }^{3}$ and Yan Xin ${ }^{4}$ \\ ${ }^{1}$ School of Information and Telecommunication Engineering, Beijing University of Posts \& Telecommunication, Beijing 100088, China \\ ${ }^{2}$ Department of Electronics Science and Technology, School of Electronics and Information Engineering, Tongji University, \\ Shanghai 201804, China \\ ${ }^{3}$ Alexander Technological Educational Institution (TEI) of Thessaloniki, 57400 Thessaloniki, Greece \\ ${ }^{4}$ Samsung Dallas Technology Labs, Dallas, TX 75082, USA
}

Correspondence should be addressed to Kan Zheng; zkan@bupt.edu.cn

Received 26 August 2014; Accepted 26 August 2014; Published 22 December 2014

Copyright (C) 2014 Kan Zheng et al. This is an open access article distributed under the Creative Commons Attribution License, which permits unrestricted use, distribution, and reproduction in any medium, provided the original work is properly cited.

Massive MIMO provides an effective means to significantly increase the capacity of wireless communication systems while possibly reducing their energy consumption. Not only the academic but also the industry has paid enough attention to the advances in massive MIMO including theory, systems, and implementation. Knowledge and understanding of the large antenna array design and realistic propagation environments may well lead to the system design and development of massive MIMO networks. To accelerate the application of massive MIMO techniques, a number of important issues must be addressed such as channel measurement, propagation and channel modeling, and efficient signal processing algorithms for massive MIMO. In this special issue on massive MIMO antenna design, channel modeling, and system technologies, we have invited a few papers that address such issues.

One of the papers of this special issue addresses raylaunching based channel models for indoor massive MIMO environments. With introducing the deterministic and statistical models, the simplified ones are proposed as well. Analysis and comparison are also given to show the effectiveness of these ray-launching based models. In one of the papers, the authors investigate the uplink performance of massive MIMO systems employing cooperative base station (BS). In their analysis, the effects of both large-scale and small-scale fading are considered as well as the spatial correlation and indoorto-outdoor high-rise propagation environment. The derived low bounds are in line with simulation results.
One of the papers of this special issue presents the survey of channel models for massive MIMO systems. The antenna array configurations are classified firstly before giving the measurement results in this paper. The channel models of massive MIMO are well investigated under different antenna array configurations. Pilot contamination is one of the great challenges in massive MIMO systems. So, in one of the papers, the authors proposed a channel estimation scheme to deal with it by utilizing interference cancellation and joint processing. The good tradeoff between the effectiveness and efficiency may be achieved by this scheme.

One of the papers focuses on how to improve the energy efficiency performance in massive MIMO systems, which is also a very important topic in this area. It proposed the power allocation scheme for the multiple pairs of nodes at both the source nodes and the relay node, where the relay employs the backward and forward zero-forcing filters. Their results demonstrated that the proposed scheme can much increase the energy efficiency while guaranteeing spectrum efficiency.

Kan Zheng

Xuefeng Yin

Periklis Chatzimisios

Yan Xin 

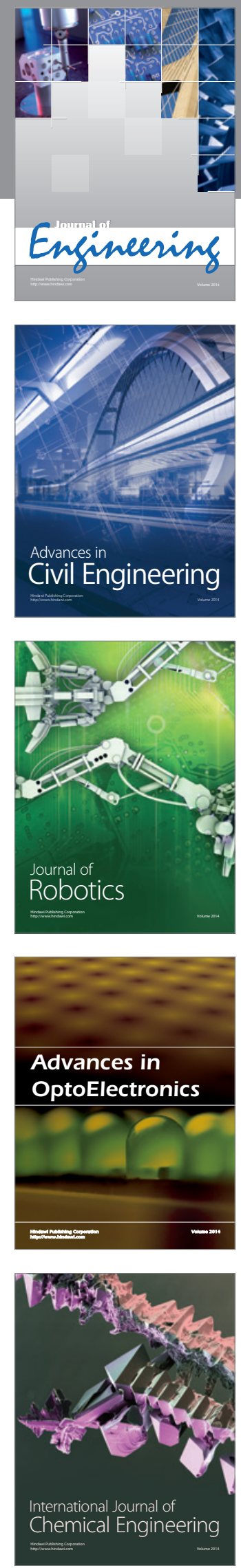

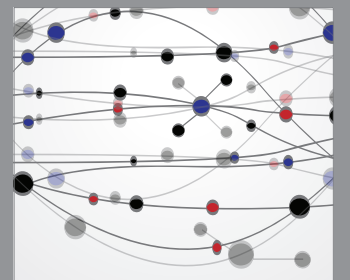

The Scientific World Journal
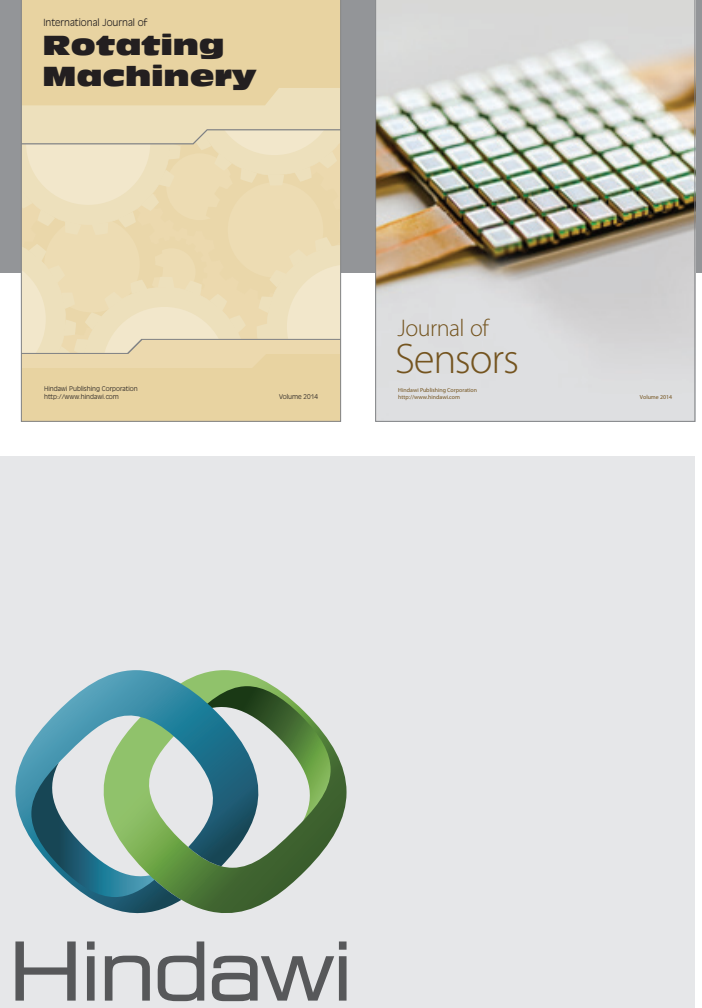

Submit your manuscripts at http://www.hindawi.com
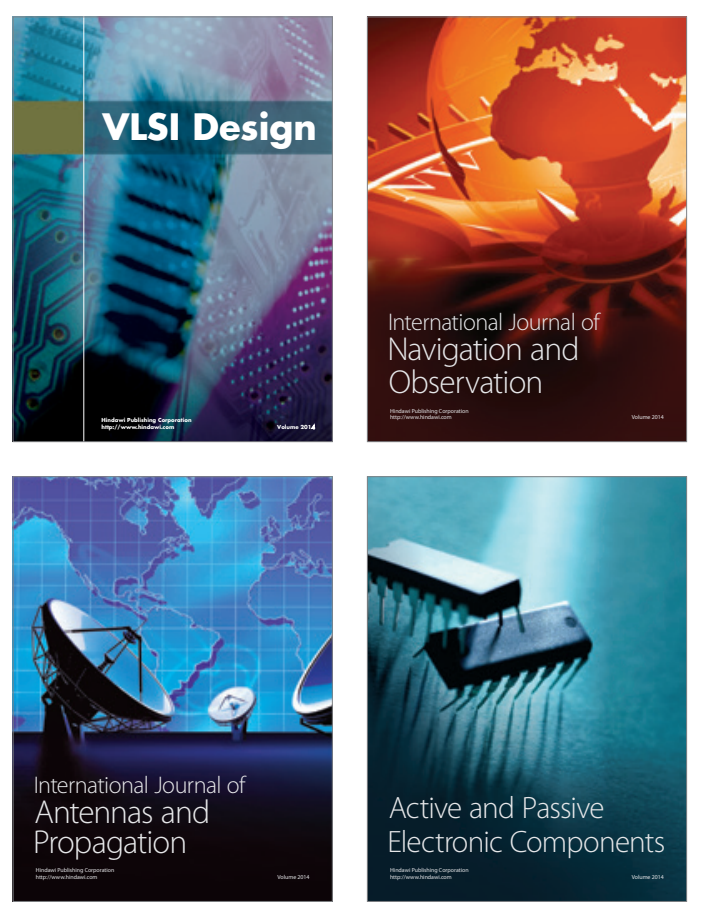
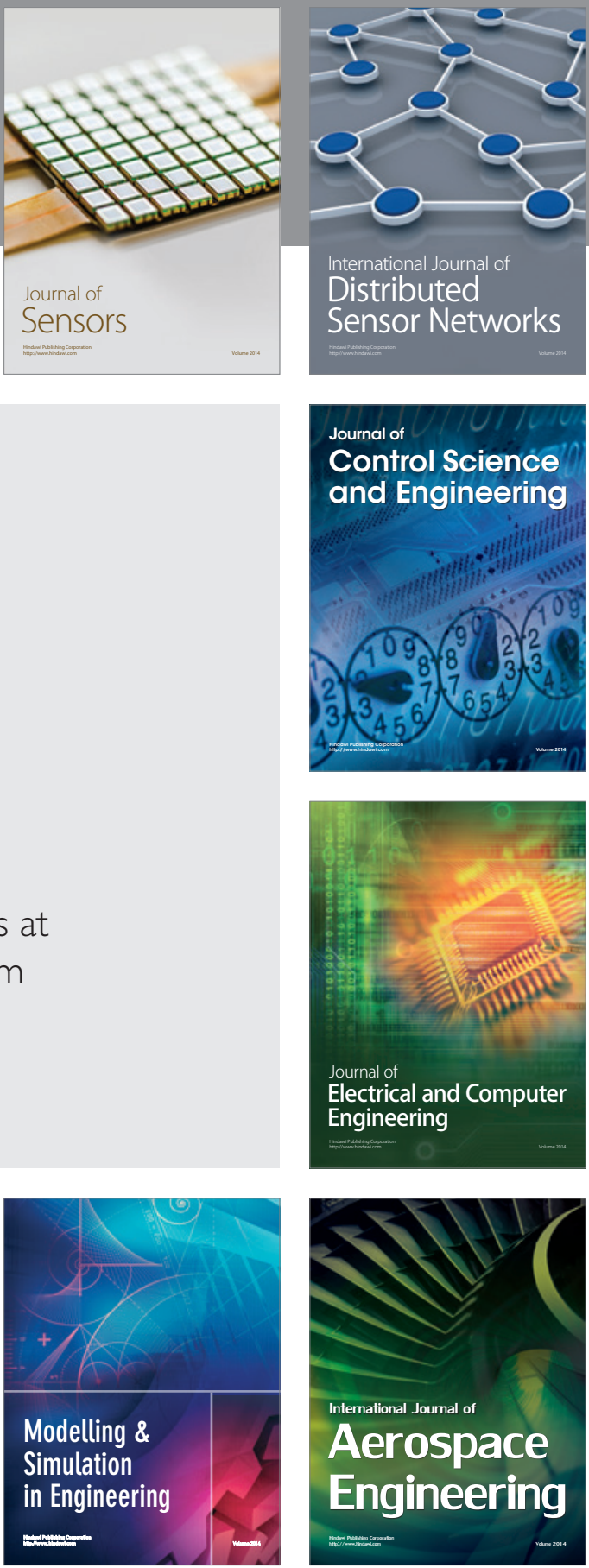

Journal of

Control Science

and Engineering
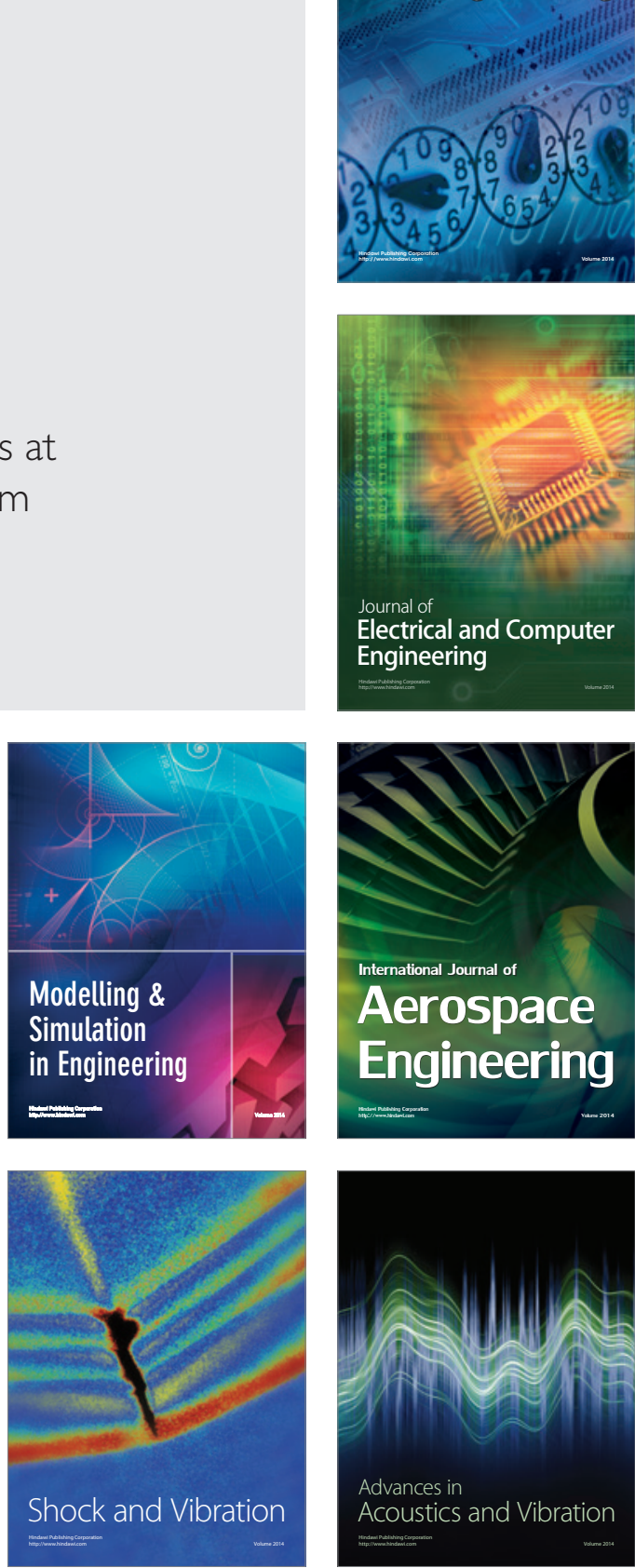